\title{
A comparison of three modelling approaches for quantitative risk assessment using the case study of Salmonella spp. in poultry meat
}

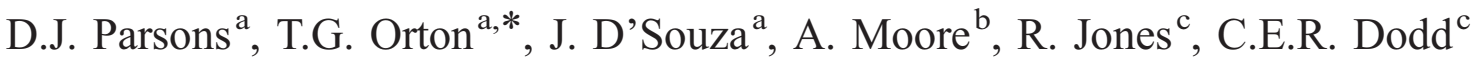 \\ ${ }^{a}$ Biomathematics Group, Silsoe Research Institute, Wrest Park, Silsoe, Bedford MK45 4HS, UK \\ ${ }^{\mathrm{b}}$ Direct Laboratories, Woodthorne, Wergs Road, Wolverhampton WV6 8TQ, UK \\ ${ }^{\mathrm{c}}$ Division of Food Sciences, Sutton Bonington Campus, University of Nottingham, Loughborough LE12 5RD, UK
}

Received 26 November 2003; received in revised form 3 May 2004; accepted 3 May 2004

\begin{abstract}
A comprehensive review of both the scientific literature and industry practices was undertaken to identify and quantify all sources of contamination throughout the entire poultry meat production chain by Salmonella spp. This information was used to develop a quantitative risk assessment (QRA) model for Salmonella in the production chain from the breeder farm to the chilled carcass. This was subsequently used as the basis on which to compare the merits of three approaches to QRA modelling in such systems. The original model used a Bayesian Network (BN). The second method was a Markov chain Monte Carlo (MCMC) approach, a numerical Bayesian technique which retained a similar network structure but allowed further development, such as the separation of variability and uncertainty. The third method was a more detailed simulation model.

The BN responds immediately to changes, such as entering evidence, because it does not use simulation and can propagate information from any point in the network to all others by Bayesian inference. However, it requires all the variables to be discrete, which introduces errors if continuous variables have to be discretized. These errors can accumulate. The MCMC approach does not require discrete variables while retaining some of the properties of the $\mathrm{BN}$ model, such as the ability to draw inferences from evidence. Finally, the simulation offers greater flexibility, such as consideration of the individual carcass, but may be more complex to implement as a result and sacrifices the ability to propagate evidence.
\end{abstract}

(C) 2004 Elsevier B.V. All rights reserved.

Keywords: Quantitative risk assessment models; Salmonella spp.; Bayesian networks; Monte Carlo methods; Poultry meat

\section{Introduction}

In 1998-1999, a 1-year project was conducted to produce a comprehensive review of both the scientific literature and industry practices and identify and quantify all sources of contamination throughout the

\footnotetext{
* Corresponding author. Tel.: +44-1525-860000; fax: +441525-861697.

E-mail address: thomas.orton@bbsrc.ac.uk (T.G. Orton).
}

entire poultry meat production chain by Salmonella spp. The review also collated information on control measures and their effectiveness for this pathogen, utilising quantitative information wherever possible. This was used to develop an initial quantitative risk assessment (QRA) model of the production chain. The review found 906 publications on Salmonella from 1988 to 1998 , of which 94 contained potentially useful quantitative data. On further examination, only about one-third could be used for model development; 
most of the others did not provide sufficient information on the effects of processes on the chain. In general, processing, especially operations such as carcass washing, received more attention than primary production.

The industry survey, which included representatives of all the large UK producers of chicken meat, found that there was widespread monitoring for Salmonella at all stages of production and implementation of HACCP procedures to control it. At that time, the contamination rate in final products was $3-20 \%$, mainly in two clusters at the ends of that range. Since then, there has been progress in reducing these levels. The model was based on the information available at the time. WHO/FAO (2002) contains surveys of more recently published information on Salmonella in poultry.

The remainder of this paper will concentrate on the QRA model for Salmonella in the poultry meat production chain from the breeder farm to the chiller. It will outline the analysis methods leading to the qualitative model and describe three approaches to the quantitative model in order to compare their strengths and weaknesses for this type of work. The first-a Bayesian network $(\mathrm{BN})$ or causal probability network (CPN) - was developed for the original study; the other two resulted from subsequent research on appropriate modelling techniques.

QRA is the study of decisions subject to uncertain consequences using the tools and techniques of probability theory and statistics (Royal Society, 1992). It is applied in many different fields; Hoornstra and Notermans (2001) looked at how to apply QRA to food safety. The typical products of a QRA exercise would be a series of statements of potential harms, whether expressed as financial costs or harmful consequences to the population, and the probabilities associated with them. Typical examples include the probability of death in middle age for male and female nonsmokers and statements of the number of deaths per passenger mile or passenger journey for different types of transport. Related disciplines include: risk perception, the study of how people think about risk; risk communication, the effective transmission of information about risk; and risk management, taking decisions in uncertain situations so as to control risk to an acceptable level. Vose (1998) looked at modelling techniques and probability distributions that can be used in a Monte Carlo simulation QRA model. This paper looks at alternative ways of implementing QRA models.

One of the key features of QRA is that it attempts to look at whole systems and not at isolated parts. Each possible adverse event is followed through to its consequences, and the consequences of different adverse events can be combined. This is only possible using a quantitative approach which provides a common basis for the evaluation of risks and harms.

The final stages of transport, retailing, cooking and consumption were not within the scope of the study, so it was not possible to quantify harm in public health terms. This would require extensive studies of the handling and treatment of poultry products in domestic and commercial kitchens and the derivation of dose response functions for exposure to the pathogen; an example of a risk assessment that does this for Salmonella in chicken can be found in WHO/FAO (2002), which commences at the conclusion of slaughterhouse processing. Instead, harm was measured in terms of the carriage rate of the pathogen on the final product. Furthermore, the sampling and culturing techniques required for the pathogen of interest mean that, generally, it is not possible to estimate the number of organisms carried; the data are normally presented simply as the proportion of birds estimated to be positive, so this is the variable with which the model has to work.

\section{Systems analysis}

Although the qualitative and quantitative analysis of this section was done with the idea of a BN model in mind, it is relevant for all of the models used in this paper.

\subsection{Qualitative analysis}

The qualitative structure of the model as a network diagram was developed through a formal systems analysis procedure which was similar in many ways to the knowledge acquisition phase of an expert system development. A series of meetings was held with 'domain experts'. These people were the partners and consultants to the project with specific expertise in the poultry industry and microbiology. In the first 
phase, meetings were held with pairs of domain experts which proceeded as follows:

1. The main stages in the process were identified: Breeder Farm, Hatchery, Broiler Farm, Processor.

2. Each stage was broken down into a series of subprocesses.

3. Inputs, outputs and cross-contamination routes at each stage were identified.

4. The resulting network was reviewed for errors and omissions.

Steps 2-4 were repeated until the parties were satisfied with the result.

As a result of the information collected, the first version of the network was constructed. This was presented at a full meeting of the project team including some of the domain experts who had not been included in phase 1. All partners were given hard copies of the network to consider and were asked for their feedback. As a result of this feedback, a second phase of revisions was undertaken after which it was agreed that all of the major features of the system had been captured. Inevitably, a few disagreements remained, but these were mainly the results of differing practice within different sectors of the industry. The main steps and some detail of the final version of the network are shown in Fig. 1.

\subsection{Quantification of the model}

As noted above, the first implementation of the model was a Bayesian network, which required quantification of each stage in terms of probabilities. These formed the basis for each of the subsequent models, so the details will be described together with the first model, but some general issues will be reviewed first.

The parameters for statistical models are usually estimated from experimental work, but no single experiment has been carried out in the detail required to allow estimation of the parameters of these models. However, in network models, quantification is per-

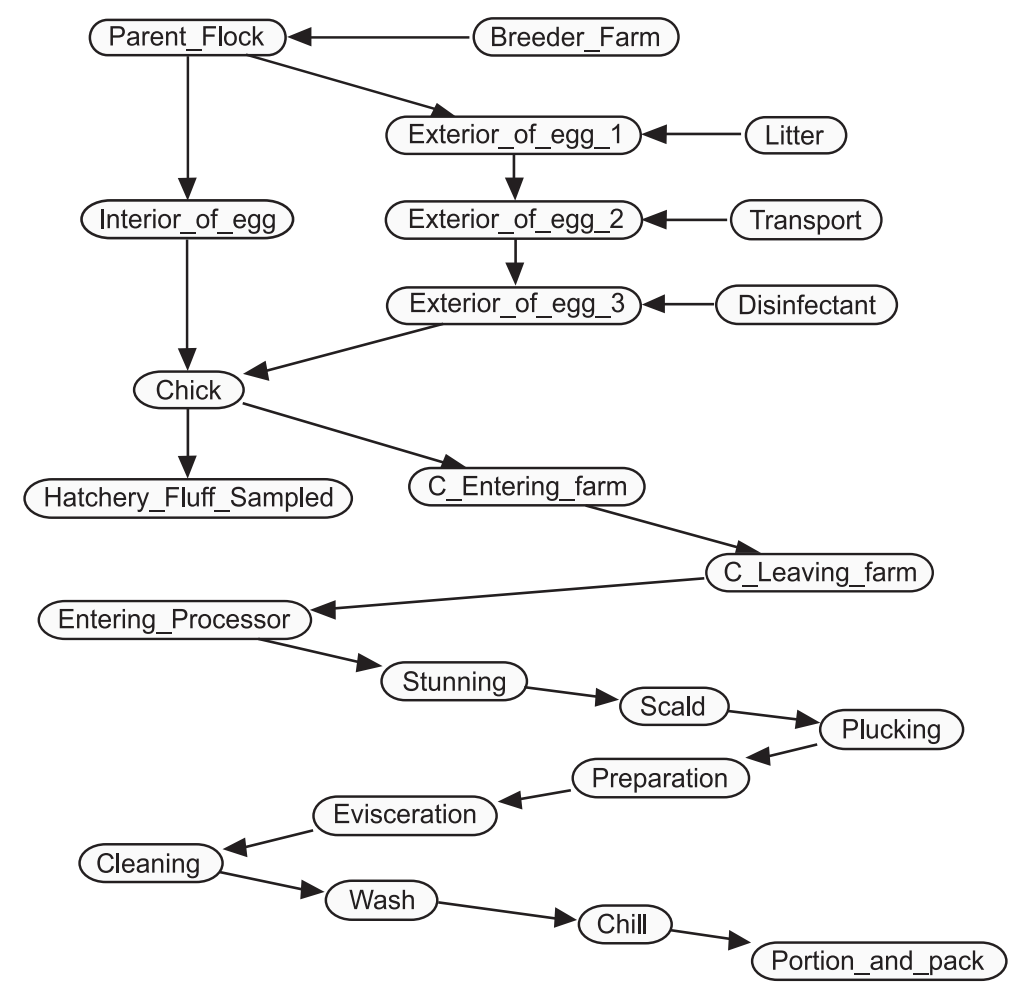

Fig. 1. Network showing the 18 prevalence nodes for the key operations and stages in the model and some of the details for the model inputs and output at the hatchery. 
formed locally for each node, so it is possible to combine data from several different sources. The Bayesian view of probability also encompasses subjective degree of belief, which extends the range of information that can be included. The sources were

1. data published in the literature where available;

2. data from the industry survey and other unpublished sources; and

3. expert opinion.

As was noted in Section 1, the literature search found about 30 papers containing data useful for the study. However, some of these could not be used to estimate model parameters directly because their data did not link processes in the model. As a result, more reliance was placed on companies' in-house data and the opinions of the expert group, and thus, the values of many parameters had to be chosen subjectively.

Edel (1994) has data showing that around 1\% of breeder flocks in The Netherlands in 1989-1992 were classed as positive. Expert opinion said that roughly $10 \%$ of the birds in a positive flock would be contaminated with Salmonella, while virtually none of the birds in a negative flock would be contaminated. The expert group thought that about $30 \%$ of the eggs laid by a contaminated bird would be positive on the interior and that there would be a strong possibility of cross-contamination of the eggshells via the litter in the nest boxes. Data from Dodd (personal communication) and the experts agree that there should only be a small change in prevalence after the transport of the eggs to the hatchery. The group disagreed about whether the disinfecting of the eggs would be $100 \%$ effective. In the model, if disinfecting is used, then $2 \%$ of the eggs that were contaminated will remain contaminated. Bailey et al. (1996) showed that about $50 \%$ of the hatched chicks will be positive when $99 \%$ of their shells are. Data from Dodd (personal communication) show that there is a low risk of crosscontamination of the chicks on transport to the farm when the level of contamination in the chicks is low. However, the quality of the disinfectant that is used in the boxes is important, and it was thought that crosscontamination can be quite high when this is poor.

On the farm, there are many factors that can influence the carriage rate of Salmonella in the flock, and various papers contain data looking at these.
Hargis et al. (1995) looked at the persistence of Salmonella enteritidis in a flock, Larsen et al. (1993) looked at the effect of treating feed, Ramirez et al. (1997) looked at the effects of feed withdrawal, Davies and Wray (1995) examined the possibility of mice carrying Salmonella and Carr et al. (1995) looked at the type of broiler shed. However, these papers do not report data that link the level on entering the farm to that on leaving the farm. The experts thought that there would be a high risk of cross-contamination on the farm. The industry survey suggested that the levels of Salmonella on entering the processor could be quite small, although data from Dodd (personal communication) show that this could be over $20 \%$. It is believed that there is a moderate risk of cross-contamination in transport to the processing plant and that the cleaning of the crates is important.

Expert opinion was divided on the effects of crosscontamination at the hanging on, stunning and bleeding stages; some said that cross-contamination would not be a problem here, while others said that there can be a high risk of aerial contamination. The risk of cross-contamination during the scalding operation could vary widely depending on the temperature of the water in the scald bath. Clouser et al. (1995) has some data showing an increase in the prevalence after the plucking operation with a conventional defeathering system, and although the sample size is small, the experts agreed that this is a major source of crosscontamination. During the head pull and preparation for evisceration, there is a high risk of cross-contamination if the viscera are exposed, which depends on good management practice.

Carramiñana et al. (1997) reported data on the changes in prevalence of Salmonella during evisceration, although no significant changes were detected. Expert opinion said that there is a high risk of crosscontamination if the guts are ruptured, but a low to moderate risk otherwise. The type of machine and setting for bird size are important factors here. The experts said that there would be a low to moderate risk of cross-contamination during the cleaning process. Various papers report data on the effects of carcass washing, but these are all in terms of $\log$ count reductions and do not report the effects on prevalence. It was thought that there would be a very low risk of cross-contamination and that the use of 
chlorine in the wash may reduce counts but not carriage rates of Salmonella. Brewer et al. (1995) looked at the effects of processing line speed on the prevalence before and after the carcass wash; they found a slight reduction in prevalence after washing at low speeds and little difference at higher speeds. Cason et al. (1997) suggested that the prevalence of Salmonella within a flock does not change much after chilling. The expert group indicated that there would be a low risk of cross-contamination, and that, like the washing, chilling may reduce counts but not carriage rates. There is a moderate to high risk of contamination and cross-contamination during the portioning and packing operations if the process is badly managed.

Having completed the initial quantification of the model, it was demonstrated to the domain experts from the project working in small groups. These meetings were used to review the final structure and the response of the model to changes in the inputs to ensure that the behaviour was reasonable. As a result of the process of quantification and review, some changes were made to the structure of the network. Several of these were simplifications made by combining consecutive processes. This was normally done when one or more processes were found to have a negligible effect and could therefore be combined with preceding or following processes whose effects were more significant. On a few occasions, processes were also combined when it was impossible to find any information on the effects of the individual processes. So, for example, the initial detailed breakdown of the series of evisceration steps was simplified to give three main steps: head pull and preparation, evisceration, and cleaning. In addition to reducing the amount of data needed, this also had the effect of reducing the number of sources of uncertainty in the model and thus the noise in the outcomes.

The other main simplification was the reduction in the number of inputs to the major processes. Initially, these had been broken down into fairly detailed categories, such as hygiene of catching crew, crate cleaning, vermin and so on. Again, there were insufficient data to be able to quantify these inputs, so in most cases, they were grouped into single nodes representing management practices and labelled in the network as 'factors'. A few of these inputs for which better data were available, for example, the scald tank temperature, were retained. To create a fully detailed model, a series of new input nodes into the factors would be required to break them down into all of the contributing elements. However, this would require the collection of detailed and site-specific data.

\section{Bayesian network model}

\subsection{Construction}

A Bayesian network consists of a graphical representation of the structure of a model and a mathematical formulation of the relationships within it (Jensen, 1996). The graphical part of the model consists of a series of boxes known as nodes, which represent the main variables in the system. The nodes are connected by arrows known as arcs, forming a directed graph. Each arc represents a causal relationship, i.e., the node at the tail of the arrow has some effect on the one at the head. In general, there may be many arcs going into and out of each node, creating a complex network. The most important restriction is that the arcs must not create loops or cycles within the network; the resulting network is known as a directed acyclic graph (DAG). An example of a $\mathrm{BN}$ approach to QRA modelling of foodborne hazards is that of Barker et al. (2002).

Fig. 1 shows a simple top-level view of the network for the poultry production system from the breeder farm through to the end of processing. In this case, each node represents one of the following: a process within the whole production system, the state of management factors or parameters for a process, or a monitoring point. The node C_Leaving_farm, for example, represents the proportion of birds that are positive when they leave the farm. So, working backwards in the network, the previous node C_Entering_farm means the proportion of birds that are positive after transport to the farm, and the arrow linking the two represents all of the changes that take place between the two points, i.e., while the birds are on the farm. All the quantities in the network are described in terms of probabilities. Within Bayesian network software packages, these must normally express discrete distributions, except for special cases, such as the Gaussian distribution. For this 
model, the carriage rate at each stage is divided into a set of categories such as less than $1 \%, 1-3 \%, 3-5 \%$, $5-10 \%$ and so on. The quantities handled by the model are the probabilities of inclusion in these classes, so the final outcome includes the probability that less than $1 \%$ are positive and so on for each class, giving a probability distribution. If necessary, the expected number can be calculated as the mean of this distribution.

For those nodes without parents (i.e., those without links coming into them), it is necessary to supply a set of initial values or prior probabilities. So, in the simplified example above, these would be the probabilities of numbers of positive birds in the parent flock. In the full model, there are many more such nodes, generally related to management practices that have an influence on the outcome.

Each of the other nodes contains a conditional probability table that expresses the relationship between the output variable from the node and the input variables. Taking the Entering_Processor node in Fig. 1 , this would be a whole array of probabilities such as the probability that less than $1 \%$ of birds entering the processor are positive given that less than $1 \%$ of birds leaving the farm are positive, or that between $1 \%$ and $3 \%$ are positive or $3 \%$ and $5 \%$ and so on. For more complex nodes, where there are several parents having a causal effect, it is easy to see that it would be necessary to create a very large table. The final model consists of 95 nodes and 121 arcs with a total of 13,414 conditional probabilities. Clearly, estimating these directly would require a very extensive data set, which is not available. However, by describing the distributions as parametric Beta distributions, the model was reduced to 60 parameters.

The distinctive feature of Bayesian Networks is that the state of every node in the network is calculated by inference using repeated applications of Bayes' rule. The initial state of the network is the joint probability distribution of all the nodes and is derived from the prior distributions of the nodes without parents and the conditional probability tables of those with. The distributions of the network in this state are called the prior distributions. Evidence may be entered into the network at any node-for example, an observation that the carriage rate on leaving the farm is between $1 \%$ and $2 \%$-and the consequences of this are propagated to all the other nodes.
With modern software and hardware, this is normally very quick, unless the network is highly connected or the conditional probability tables are very large. The resulting distributions are called the posterior distributions.

The model was implemented using the NETICA package produced by Norsys Software of Vancouver. The full version of the model expands on the simplified form presented in Fig. 1 and includes four types of nodes.

1. Those in the main trunk of the network represent a random variable corresponding to the level of contamination at a particular stage of the process. For example, the node Stunning represents the proportion of contaminated birds immediately after the stunning/bleeding stage.

2. Inputs which represent the level of hygiene at a certain stage (so Scald_Temp flags whether scald is hard or soft); the management factors nodes are of this type.

3. Outputs, such as Hatchery_Fluff_Sampled, representing a quantity that could be measured.

4. Model parameters (e.g., T1_Scald, T2_Scald, Beta_Scald and P_Scald)

These are illustrated in Figs. 1 and 2. Each of the nodes in the main trunk, such as Scald, represents the prevalence distribution of Salmonella in the flock defined numerically as a Beta distribution conditional on the values of the node's parents. Because the Beta distribution is continuous, this is divided into series of discrete categories as described earlier. The model parameters for any stage, $P, T 1, T 2$ and Beta are used to derive the parameters of this Beta distribution. The parameter Beta may be interpreted as a measure of the variability in the data; large values of Beta correspond to small variances. $T 1$ measures the probability that an egg, bird or carcass that is contaminated before a particular process will still be contaminated at the end of it. In general, this value is close to 1 as very few processes actually reduce contamination. $T 2$ is a measure of the probability that an uncontaminated bird will become contaminated and is normally small but very rarely zero. Finally, $P$ measures the relative importance of environmental contamination and cross-contamination. If cross-contamination is not a major problem in a process, then $P$ will take a large 


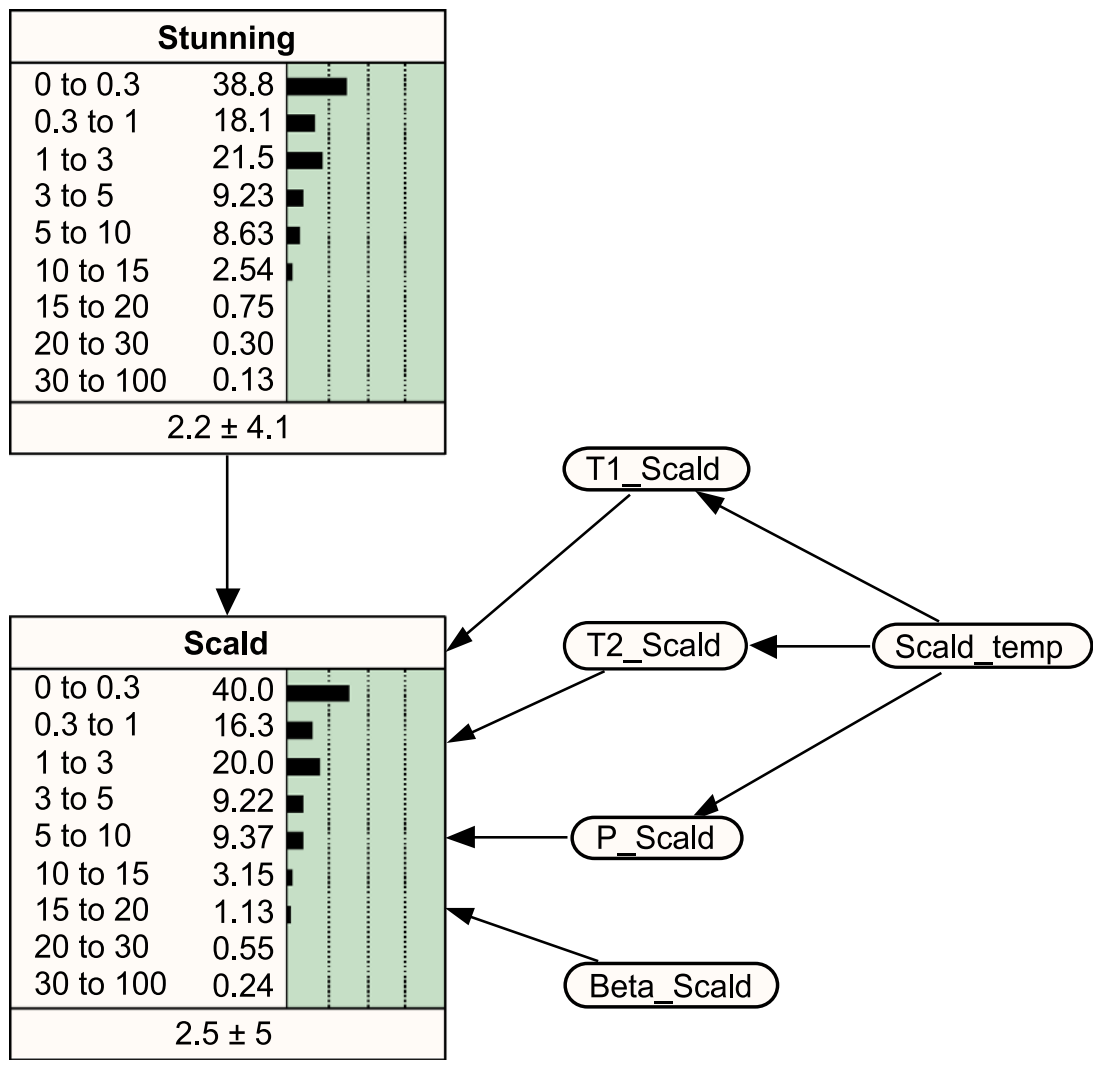

Fig. 2. The scald tank section of the full network showing the stage specific factors (Scald_temp) and parameter nodes (T1_Scald, T2_Scald, P_Scald, Beta_Scald) and prevalence nodes (Stunning and Scald) before and after the scalding process.

value, whereas if cross-contamination is dominant, $P$ will have a small value.

\subsection{Testing}

The absence of suitable data for development of the model also meant that there were no suitable data sets for validation. The best that could be done was to look at the general outcomes in comparison with sources such as the survey data and to look at the responses to specific changes in the input variables.

With no inputs set, the 'factors' have their default distributions (typically Prob[good] $=0.01$, Prob[average $]=0.98$, Prob[poor $]=0.01$ ). All of the nodes in the model then have their prior distributions. The prior distribution of the final carriage rate (shown in Fig. $3 \mathrm{~A}$ ) had a mean of $8 \%$. With all the factors set to 'good' or the equivalent, the mean of this final carriage rate was reduced to $4 \%$. The effects on the mean of changing individual inputs from the default to the worst setting are shown in Table 1 .

The range of responses is plausible in the light of the survey result described earlier, where the best producers achieved mean final carriage rates of $3-$ $5 \%$, but poorer controls led to rates in the range of $13-23 \%$. The relative effects of the different factors are also reasonable, although the absolute values require confirmation.

\subsection{Results}

The model can be used to make inferences about system variables. Setting certain prevalence nodes to be 'observed', the effects of this evidence are propagated throughout the network, and the updated marginal distributions of all other domain variables can be viewed. For instance, should a flock of poultry arriv- 
A

\begin{tabular}{|c|c|c|}
\hline \multicolumn{3}{|c|}{ Parent_Flock } \\
\hline 0 to 0.2 & 80.7 & \\
\hline 0.2 to 1 & ש & \\
\hline 1 to 3 & 3.44 & \\
\hline 3 to 5 & 0.46 & \\
\hline 5 to 10 & 0.69 & \\
\hline 10 to 15 & 0.44 & \\
\hline 15 to 20 & 0.23 & \\
\hline 20 to 30 & 0.14 & \\
\hline 30 to 100 & .018 & \\
\hline \multicolumn{3}{|c|}{$0.4 \pm 1.9$} \\
\hline \multicolumn{3}{|c|}{$\downarrow$} \\
\hline \multicolumn{3}{|c|}{ Entering_Processor } \\
\hline 0 to 0.3 & 40.5 & \\
\hline 0.3 to 1 & $18.8=$ & \\
\hline 1 to 3 & 21.7 & \\
\hline 3 to 5 & 8.85 & \\
\hline 5 to 10 & 7.50 & \\
\hline 10 to 15 & 1.88 & \\
\hline 15 to 20 & 0.48 & \\
\hline 20 to 30 & 0.18 & \\
\hline 30 to 100 & .085 & \\
\hline \multicolumn{3}{|c|}{$2 \pm 3.6$} \\
\hline & $\downarrow$ & \\
\hline \multicolumn{3}{|c|}{ Portion_and_pack } \\
\hline 0 to 0.5 & 20.2 & \\
\hline 0.5 to 1 & 12.2 & \\
\hline 1 to 2 & 15.6 & \\
\hline 2 to 3 & 9.25 & \\
\hline 3 to 5 & 10.3 & \\
\hline 5 to 10 & 12.3 & \\
\hline 10 to 15 & 6.66 & \\
\hline $\begin{array}{l}15 \text { to } 20 \\
20 \text { to } 30\end{array}$ & $\begin{array}{l}4.04 \\
4.05\end{array}$ & \\
\hline 30 to 100 & 5.40 & \\
\hline & \pm 16 & \\
\hline
\end{tabular}

B

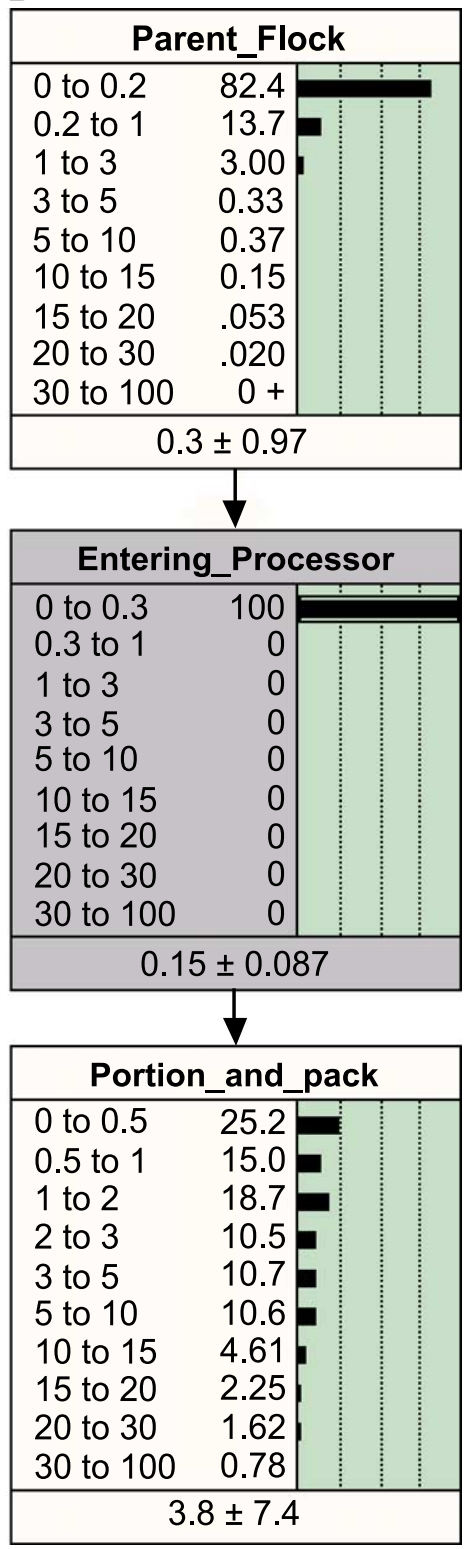

Fig. 3. The prior (A) and posterior (with the evidence that the prevalence on entering the processor is less than $0.3 \%$; B) marginal distributions for the prevalence nodes Parent_Flock, Entering_Processor and Portion_and_Pack in the original BN model. The arrows show the causal links with all of the intermediate nodes absorbed. Each distribution's mean and standard deviation is displayed at the bottom of the node.

ing at the processing plant be observed to have a prevalence of less than $0.3 \%$, this information could be entered into the network and the likely causes and effects viewed in the form of updated 'posterior' probability distributions. Fig. 3 shows the prior (be- fore this evidence is entered into the network) and posterior distributions for the nodes Parent_Flock, Entering_Processor and Portion_and_Pack.

Systematically progressing through the network and investigating the effects of changing poor factors 
Table 1

The effect on the mean final prevalence in the BN model of setting individual input factors to 'poor'

\begin{tabular}{lc}
\hline Factor & Prevalence (\%) \\
\hline Default settings & 8 \\
Breeder_Farm = Positive & 16 \\
Disinfectant (egg) = No & 18 \\
Factors_Chick_Leaving_Farm = Poor & 16 \\
Factors_Stunning = Poor & 10 \\
Scald_Temperature $<$ 55C & 15 \\
Factors_Plucking = Poor & 11 \\
Factors_Evisceration= Poor & 11 \\
Percentage_Chlorine= Absent & 9 \\
Factors_Portion_and_Pack=Poor & 13 \\
\hline
\end{tabular}

to good showed that in general, contamination early in the process produces the largest effect. However, the final portion-and-pack operation, with high potential for contamination and cross-contamination, is also important. Clearly, multiple failures of hygiene will lead to higher carriage rates. On the other hand, disinfection of the eggshell (usually with formaldehyde) can control problems caused by external contamination of eggs. Setting the hatchery litter condition to 'bad' without disinfection produces a mean final carriage rate of $26 \%$ through infection of the chicks. This is returned to $8 \%$ by the use of disinfectant.

One useful feature of the Bayesian Network modelling approach is that it allows the inclusion of outcome nodes representing sampling points. Such nodes are normally linked to the model variables by tables representing the probabilities of having true or false positive and true or false negative observations. They can thus model the uncertainty that is present in observations and be used to investigate the potential benefits of improved sampling and assay techniques. At present, the only such node is Hatchery_Fluff_ Sampled, which represents the outcome of testing a fluff sample for the presence of Salmonella. With the default settings, the outcome here is $1.3 \%$ of samples positive. Setting the sample to negative had a negligible effect on the final carriage rate, but setting it to positive increased the mean rate to $24 \%$ because it implied a serious hygiene failure in the hatchery or breeder farm. Although this result is only illustrative, it shows how the use of monitoring points can be modelled.

\subsection{Discussion}

The network approach was used as it is an excellent probabilistic reasoning tool. The local quantification of variables enables the parameter specification process to incorporate data from various sources and the opinions of field experts. By manipulating the users' assumptions of conditional independence, the BN software updates the probability distributions for all other domain variables in the presence of observations of certain variables, allowing the user to make inferences and answer questions about the interactions between variables in the system. However, because the $\mathrm{BN}$ method requires the discretization of general continuous variables, errors can result in the marginal distributions.

\section{MCMC models}

\subsection{Construction}

The Bayesian methodology seems appropriate for modelling the prevalence of Salmonella-positive birds in a flock, with its approach to uncertainty and its ability to infer about both likely causes and effects of observations. The BN method used, however, relies on the discretization of continuous variables, and this incurs errors in the marginal distributions. Markov chain Monte Carlo (MCMC) methods provide an alternative means of updating the probabilities in a network model. They use numerical integration to approximate probability distributions and therefore do not necessitate the division of continuous variables into discrete ranges.

The WinBUGS software package can be used for the Bayesian analysis of complex statistical models using MCMC techniques (Spiegelhalter et al., 2003). It allows users to set up a model as a DAG, incorporating both stochastic and deterministic nodes. The system is given some consistent initial state and the program then progresses through some causal ordering of the nodes, sampling from each of their conditional distributions (given the current values of all other variables in the system). When this process is repeated (using the same ordering of nodes), the distributions produced converge to the true marginal probability distributions. For an introduction to 
MCMC methods and their applications, see Brooks (1998).

A model was constructed in WinBUGS as an equivalent of the $\mathrm{BN}$ model. The only difference between the models produced using the two approaches was that the $\mathrm{BN}$ model required that the continuous variables be discretized, whereas the MCMC model did not. Using an MCMC sampling algorithm, the resulting distributions converge to the target densities. This produces substantially different marginal distributions (particularly for 'downstream' nodes) from those produced in the Netica BN software. These differences in the output arise due to the discretization of continuous variables, as is required by the BN approach. Denoting the true target density by $\pi$ and by taking a large enough sample, MCMC methods such as the Gibbs sampler can be used to get as close an approximation to $\pi$ as desired, and thus, this approach seems more reliable in this example.

A method for determining the number of iterations, $n$, required for convergence of one long run of the Gibbs sampler can be found in Brooks (1998). This method produces an estimate of $n=27,000$ iterations for convergence of the Portion_and_Pack distribution in the model. This is based on a pilot run of 1000 iterations. It produces $n$ for which there is a probability of 0.95 that the mean of these samples lies within 0.001 of the true mean. Similar calculations for the other variables and models used in this section all suggest that using 50,000 iterations is sufficient to be confident of convergence.

\subsection{Results}

The WinBUGS program was used to produce 50,000 iterates of the model. This resulted in the marginal distributions for the prevalence in the parent flock, the flock on entering the processor and the processed and packaged carcasses, shown as a table of summary statistics in Table 2, and as smoothed density plots in Fig. 4A. To show how these distributions might be compared to those produced using the $\mathrm{BN}$ model, the prior distribution for the final portion-and-pack operation is discretized using the same range boundaries as in the BN model and this is shown in Fig. 5. The two different causal probability network updating methods produce significantly different output in terms of the means of the final marginal distributions; the BN approach gives $\mu=8 \%$ and the MCMC approach gives $\mu=4 \%$.

As with the BN model, inferences can be made about system variables using MCMC methods. This is done by entering evidence and then sampling to approximate all other posterior distributions. Although using this method for inference is slower than using a $\mathrm{BN}$ for a similar model, the answers obtained are more accurate, so long as enough samples have been taken for convergence. Using the same example evidence as entered into the BN model $(0-0.3 \%$ prevalence on entering the processor), 50,000 iterations were run. This produced the posterior distributions for the Parent_Flock, Entering_Processor and Portion_and_Pack nodes shown in Fig. 4B as smoothed density plots and in Table 2 as summary statistics. Comparing the posterior distributions produced using the MCMC method with those produced using the $\mathrm{BN}$ once again highlights the differences between the two approaches. Where the $\mathrm{BN}$ method produced a final expected carriage rate of $3.8 \%$ under this evidence, the MCMC approach suggested a lower value of $1.7 \%$. The expected prevalence in the parent

Table 2

Summary statistics for the prior and posterior (with the evidence that the prevalence on entering the processor is less than $0.3 \%$ ) prevalence distributions of Salmonella in the parent flock, the flock upon entering the processor and after portioning and packing, as given by the MCMC Beta distribution model

\begin{tabular}{|c|c|c|c|c|c|c|}
\hline \multirow[t]{2}{*}{ Node } & \multicolumn{3}{|c|}{ Prior distributions } & \multicolumn{3}{|c|}{ Posterior distributions } \\
\hline & Mean & 2.5 percentile & 97.5 percentile & Mean & 2.5 percentile & 97.5 percentile \\
\hline Parent_Flock & 0.27 & $1.510^{-15}$ & 1.8 & 0.18 & $2.110^{-19}$ & 1.2 \\
\hline Entering_Processor & 1.4 & $1.910^{-10}$ & 8.7 & 0.054 & $4.310^{-13}$ & 0.27 \\
\hline Portion_and_Pack & 4.0 & 0.0017 & 29 & 1.7 & $9.610^{-4}$ & 11 \\
\hline
\end{tabular}

This model was created as an equivalent to the original $\mathrm{BN}$ model. 

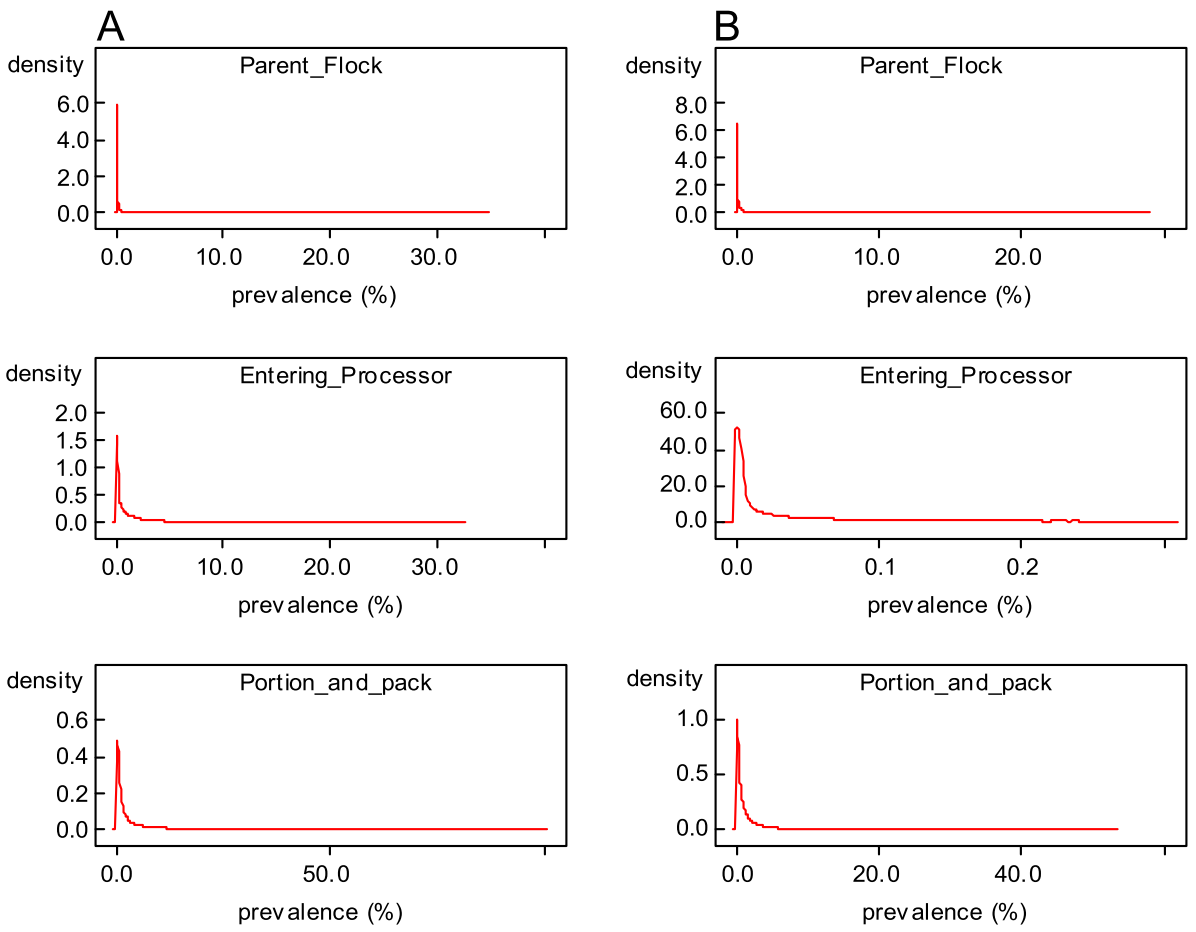

Fig. 4. The prior (A) and posterior (with the evidence that the prevalence on entering the processor is less than $0.3 \%$; B) marginal distributions for the prevalence nodes Parent_Flock, Entering_Processor and Portion_and_Pack in the MCMC Beta distribution model. This model was created as an equivalent to the original BN model.

\begin{tabular}{|ll|}
\hline \multicolumn{3}{|c|}{ Portion_and_pack } \\
\hline 0 to 0.5 & 40.2 \\
0.5 to 1 & 13.0 \\
1 to 2 & 12.7 \\
2 to 3 & 6.69 \\
3 to 5 & 7.09 \\
5 to 10 & 8.89 \\
10 to 15 & 4.31 \\
15 to 20 & 2.51 \\
20 to 30 & 2.36 \\
30 to 100 & 2.23 \\
\hline \multicolumn{3}{|c|}{$5 \pm 11$} \\
\hline
\end{tabular}

Fig. 5. The prior marginal distribution for the Portion_and_Pack node in the MCMC Beta distribution model. This model was created as an equivalent to the original $\mathrm{BN}$ model. The continuous marginal probability distribution has been discretized to show the differences that arise between the output from the two methods of calculating the marginal probability distributions with the same model. flock for the same evidence was $0.30 \%$ when the BN was used and $0.18 \%$ when MCMC methods were.

\subsection{Model development}

The MCMC model described above was formulated for comparison with the BN model, so the parameters were modelled by discrete distributions. However, with MCMC methods, one can consider modelling the parameters with continuous distributions. This is done by fitting distributions about each of the point estimates for the parameters from the BN model. Beta distributions are fitted about the $T 1$ and $T 2$ parameter estimates, and censored normal distributions, about the $P$ parameter estimates; $T 1$ and $T 2$ are therefore defined as Beta-mixture and $P$ as normal-mixture distributions.

The Beta distribution was used in the original model for the prevalence of Salmonella in the flock as a simplification; it is flexible enough to model a wide range of behaviour over a closed interval. 
However, given the parameters as defined, it is more natural to consider a combination of binomial distributions.

By considering the number of birds in a flock that are contaminated with Salmonella, it is possible to model the number of birds that remains contaminated and the number that becomes contaminated after a certain process. Both of these are modelled separately by binomial $(n, p)$ distributions, and the sum of these two variables gives the total number of birds that is contaminated with Salmonella after the process. The parameters $T 1, T 2$ and $P$, along with the number of birds previously contaminated and the flock size variable, $N$, are used to derive the relevant $n$ and $p$ parameters. The flock size variable $N$ is set to $N=10,000$ for the following models.

This model is an example of what is known as two-dimensional modelling. Vose (1998) defines uncertainty as the lack of precise knowledge one has about a model parameter and variability as the random effects of chance that might occur in the system. A two-dimensional model aspires to separate these two components, and in this case, does so by encapsulating the variability in the binomial distributions and the uncertainty in the (continuous) parameter distributions.

This two-dimensional model can be used to investigate the effects of variability and uncertainty on the output. Table 3 compares some summary statistics for the final Portion_and_Pack distribution in the different instances of this two-dimensional model. With fixed point estimates for the parameters, the resulting model is without uncertainty (as defined above) but only contains variability, and the final distribution statistics for this model are shown in row 3 of the table. With continuous parameter distributions, but a deterministic model which uses these parameters to calculate an expected number of contaminated birds at each stage, the model has uncertainty but no variability. The final marginal distribution, in this case, is spread depending on the variances of the parameter distributions (see rows 1 and 2 of Table 3).

With the state of factors known for all nodes, the model can be thought of as representing a known farm or processing plant, on which the state of the management factors for each operation can be categorised as either good, average or poor (or otherwise, as appropriate). With the discrete parameter model, this gives fixed parameter values for all stages. Row 3 of the table shows the statistics for this scenario (with all factors in their most probable state) and gives a very narrow $95 \%$ interval of $[2.6 \%$, $3.4 \%$ ]. The continuous parameter model gives a more broadly spread output, the $95 \%$ interval being [0.31\%, 13\%] (row 5 of Table 3), reflecting a more realistic scenario.

\subsection{Discussion}

MCMC methods provide a good means of approximating the marginal probabilities in a Bayesian network. The fact that it is not necessary to discretize the continuous variables means that such errors are not incurred and that the methods can be used to produce a more accurate representation of a model. The two-dimensional approach appears to be more

Table 3

Summary statistics for the prior prevalence distributions of Salmonella in the flock after portioning and packing, as given by the MCMC binomial distribution model

\begin{tabular}{lllllllc}
\hline & Uncertainty & Parameter distributions & Factors & Variability & Mean & 2.5 percentile & 97.5 percentile \\
\hline 1 & Yes & Discrete & Unknown & No & 3.3 & 2.7 & 6.3 \\
2 & Yes & Continuous & Unknown & No & 4.0 & 0.34 & 14 \\
3 & No & Discrete & Known ${ }^{\mathrm{a}}$ & Yes & 2.9 & 2.6 & 3.4 \\
4 & Yes & Discrete & Unknown & Yes & 3.2 & 2.5 & 6.6 \\
5 & Yes & Continuous & Known ${ }^{\mathrm{a}}$ & Yes & 3.6 & 0.31 & 13 \\
6 & Yes & Continuous & Unknown & Yes & 4.0 & 0.33 & 14 \\
\hline
\end{tabular}

This model is an example of two-dimensional modelling, in which the variability (binomial distributions for individual variation) and uncertainty (discrete or continuous distributions for parameters, 'factors' considered to be known-i.e., good/average/poor-or unknown) are separated out. The table shows six different combinations of models for variability and uncertainty.

${ }^{\text {a }}$ Factors in most probable state. 
transparent than the original model, which combined both uncertainty and variability in simplifying the situation to fit within the $\mathrm{BN}$ framework. Considering such a two-dimensional model within the BN framework would require the introduction of further continuous nodes. The attempt to separate out the uncertainty from the variability would introduce more discretization errors to the network.

\section{Simulation models}

\subsection{Construction}

Another approach that may be used to model the contamination of poultry with Salmonella spp. is Monte Carlo simulation. This uses forward sampling and has no reliance on Bayes' law and on the calculation of conditional sampling distributions. It can be used to more efficiently tackle some problems than the Bayesian methods could and hence to model more complex interactions between domain variables. An example of a QRA model that uses this methodology is by Cassin et al. (1998). This uses Monte Carlo simulation to assess the human health risk associated with Escherichia coli $\mathrm{O} 157: \mathrm{H} 7$ in ground beef hamburgers.

A computer program was written, using the $\mathrm{C}++$ programming language, which modelled the status of individual birds throughout the system. For each stage of the example system, each bird was considered separately as a Bernoulli trial with a probability dependent on its own current status, on the current prevalence in the flock and on the stage parameters. The two-dimensional modelling approach was used with both uncertainty and variability included. The parameters were modelled by continuous distributions and the original default distributions were used for the states of the factors. This model was essentially the same as the two-dimensional MCMC model but considered the birds in separate Bernoulli trials as opposed to together in a binomial distribution.

It should be noted here that the choice to write a program from scratch rather than use an existing package for the simulation was made after brief trials with the @RISK (Palisade) package. The simulation models the status of 10,000 birds as they pass through the various stages and operations. The status of each these birds at each of these operations is a random variable, giving 180,000 variables, too many for an Excel (Microsoft) spreadsheet to handle. In addition, the package was found to be slow for the kind of simulation being performed, which consists of a chain of distributions, each deriving its parameters from samples from the previous distribution in the chain.

\subsection{Results}

The model was run for 50,000 iterations. The mean and $95 \%$ interval of the resulting marginal distributions for the prevalence in the parent flock, on entering the processing plant and after portioning and packing are shown in Table 4. These summary statistics are, as one might expect, similar to those arrived at for the binomial model updated using MCMC methods (see Table 3, row 6). The kernel density estimates are shown in Fig. 6A.

Suppose that the system is to be studied under the observation of a prevalence of between $0 \%$ and $0.3 \%$ on entering the processor. Then the program is run as before, retaining only the iterations of the sampler for which this condition is satisfied. This leads to approx-

Table 4

Summary statistics for the prior and posterior (with the evidence that the prevalence on entering the processor is less than $0.3 \%$ ) prevalence distributions of Salmonella in the parent flock, the flock upon entering the processor and after portioning and packing, as given by the first version of the MC simulation model

\begin{tabular}{|c|c|c|c|c|c|c|}
\hline \multirow[t]{2}{*}{ Node } & \multicolumn{3}{|c|}{ Prior distributions } & \multicolumn{3}{|c|}{ Posterior distributions } \\
\hline & Mean & 2.5 percentile & 97.5 percentile & Mean & 2.5 percentile & 97.5 percentile \\
\hline Parent_Flock & 0.30 & 0 & 2.0 & 0.25 & 0 & 1.7 \\
\hline Entering_Processor & 1.4 & 0.04 & 6.3 & 0.15 & 0.02 & 0.3 \\
\hline Portion_and_Pack & 3.8 & 0.24 & 15 & 1.8 & 0.11 & 7.5 \\
\hline
\end{tabular}

This model uses the parameters defined for the original BN model but considers the individual birds separately rather than the flock as a whole. 

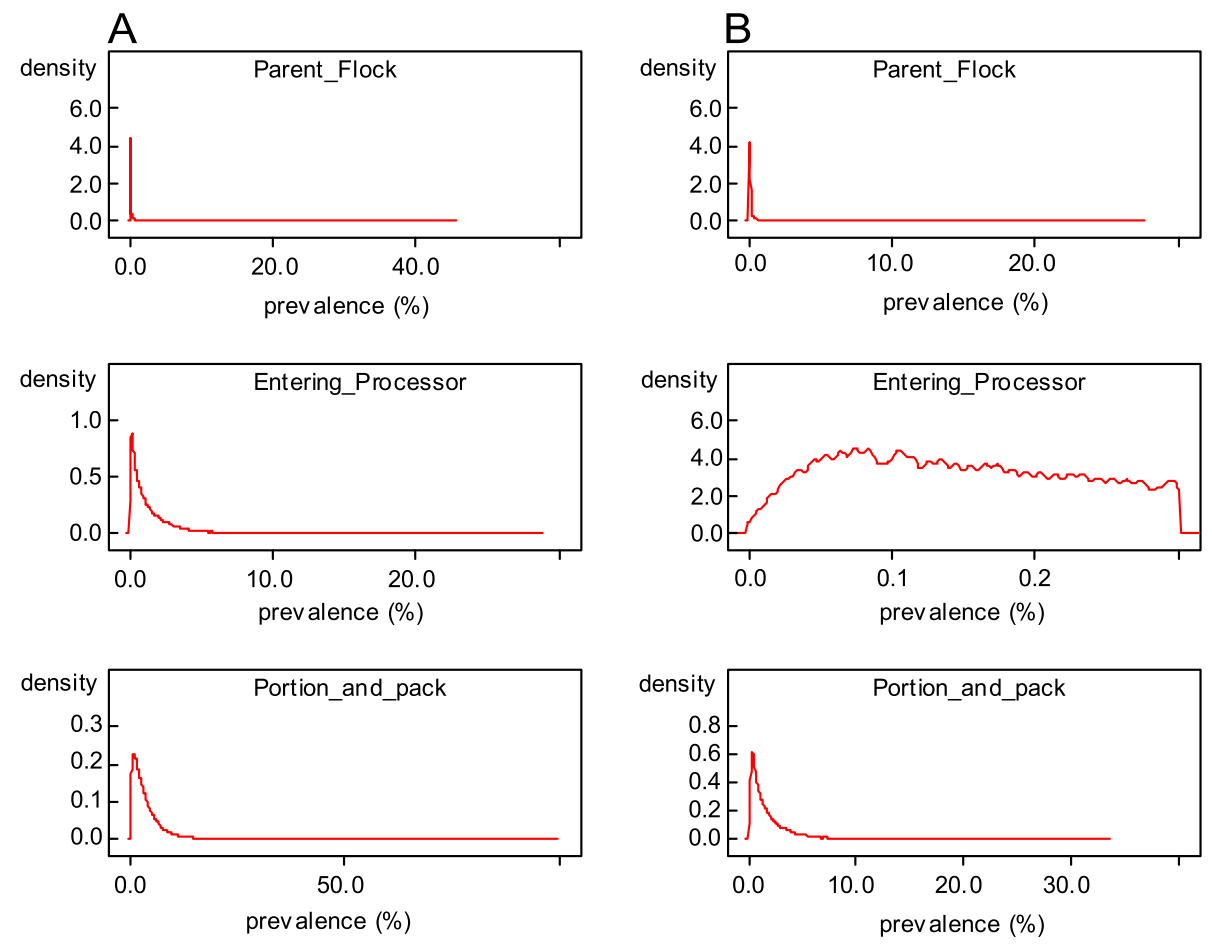

Fig. 6. The prior (A) and posterior (with the evidence that the prevalence on entering the processor is less than $0.3 \%$; B) marginal distributions for the variables Parent_Flock, Entering_Processor and Portion_and_Pack in the first version of the MC simulation model. This model uses the parameters defined for the original $\mathrm{BN}$ model but considers the individual birds separately rather than the flock as a whole.

imately 12,500 of the 50,000 iterations being analysed, discarding three-quarters of the iterations performed. Fig. 6B shows the kernel density estimates for the variables Parent_Flock, Entering_Processor and Portion_and_Pack, and Table 4, the summary statistics. The results of these inferences are comparable to those made with previous models (see Fig. 3 and Table 2); the effect of the evidence on the means of the marginal distributions is a similar reduction in all models.

\subsection{Model development}

Using the Monte Carlo simulation approach, a more complex model can be considered than would be practical in a Bayesian methodology. Suppose that there is a flock of birds entering the processing plant. These birds are immediately hung on shackles on a conveyor to be stunned. Once on the conveyor, the individual birds can be considered ordered in a sequence, in which it seems plausible that for each bird, cross-contamination is more likely to come from the birds in a close proximity to it than from those further away. In the previous models, the cross-contamination depended on the prevalence of Salmonella within the entire flock. With the consideration of the individual bird or carcass, this could be made to depend on the status of the surrounding birds only. A weighted average of the previous $k$ birds to pass through was used to determine the cross-contamination probability, where $k$ was chosen to be 100 .

Using the two-dimensional model with both variability and uncertainty, 50,000 iterations of the forward sampling algorithm were performed. This produced the distribution for the prevalence after the portioning and packing operation shown as a smoothed probability density estimate in Fig. 7. This distribution has mean $4.1 \%$ and $95 \%$ interval $[0.26 \%$, $15 \%]$. The distributions for the prevalence in the 


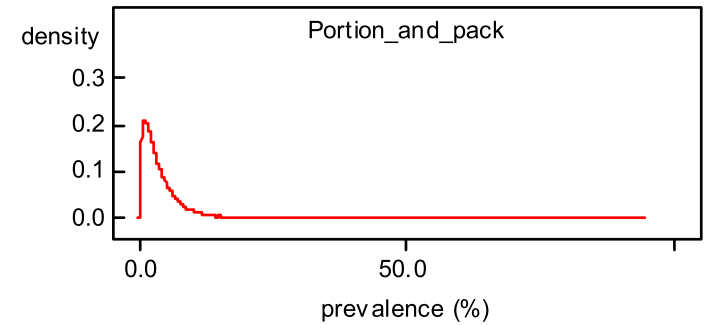

Fig. 7. The marginal distribution for the prevalence of Salmonella in the portioned and packaged carcasses from the second version of the MC simulation model. This model considers that for any bird, crosscontamination is dependent only on the birds immediately surrounding that bird and not on the prevalence within the entire flock.

parent flock and on entering the processing plant are identical to those of the previous Monte Carlo simulation model (because the only changes concern stages from entering the processor onwards).

\subsection{Discussion}

The model developed in this section is an example intended to illustrate some of the possibilities offered by Monte Carlo simulation, and further extensions of this could of course be investigated. Although it would be possible to use the Bayesian methodology in formulating a similar model, the additional complexity might render it impractical.

In a full microbiological risk assessment, it is normally necessary not only to model the prevalence of positive birds in a flock but also to quantify the number of organisms present in these birds. This is done so that dose-response studies may be incorporated into the QRA, and the associated human health risks investigated. In order to do this, it appears sensible to consider an individual based model, which may be more suited to the simulation framework, as the problem could be too complex to consider a model in the Bayesian format. An example of such a model that looks at the numbers of organisms present is by Whiting and Buchanan (1997) for S. enteritidis in pasteurised liquid eggs. However, lack of relevant data that quantifies the number of organisms present at the various stages in the model can often mean that a complete quantitative model is not possible, and so the benefits offered by a Monte Carlo simulation may be redundant.

\section{Discussion}

In this paper, it has been shown how the techniques of Bayesian network modelling, Markov chain Monte Carlo and simulation modelling can be used to formulate a model for use in a quantitative risk assessment, within the context of one particular example system. The potential of each method has been illustrated by considering various different types of model.

The BN method was found not to represent the heavily skewed distributions accurately due to the need to discretize continuous variables. In the example network, these errors are compounded throughout the system, so that the mean of the final prevalence distribution was given as $8 \%$, when the true representation of this model should be around 4\%. Although it is a quick and straightforward task to enter evidence into the network and make inferences about other domain variables, the answers produced can be inaccurate, as was shown in the example.

Markov chain Monte Carlo methods can be used to produce an approximation to the target distribution, and this, in the models for the Salmonella example, proved to be a more accurate way of updating the probabilities than the $\mathrm{BN}$ method. As the number of samples taken is increased, this approximation converges, and the number of iterations required can be estimated. Inferences, in the light of evidence, can be made on all domain variables using MCMC methods. This is done by entering the observations (i.e., fixing the values of the appropriate variables) and performing iterations of the sampling algorithm until convergence occurs. Because MCMC methods deal directly with the continuous variables in a system, such methods may be confidently used to approximate the marginal probabilities in twodimensional models, which separate out the uncertainty and variability.

The simulation method offers more modelling flexibility. Because it only considers forward sampling, there is no need for any calculation of complicated conditional distributions (using Bayes' law), and thus more complex models may be considered. This allows the effects of introducing logical expressions to the model, dependent on many other variables, to be investigated, and this also enables models to look at both prevalence and the load of an organism in a QRA. Were a DAG to be considered for such a model, 
it would have to be highly connected, and the sampling procedure rendered painfully slow.

Inference in the simulation framework is not so straightforward. Analysis of the system when there is an observation of some variable is performed by running many simulations of the model and retaining only the runs for which this condition is satisfied. However, if the probability of this is small, then this process will be very wasteful and time consuming. Worse still, should the evidence be an observation of a point value for a continuous variable, this approach to inference becomes impossible.

\section{Conclusions}

The Bayesian network method was shown to be the most appropriate when inference and speed are important. However, complexity is limited and errors due to the discretization of continuous variables can render this method an inaccurate representation of the model distributions.

Monte Carlo simulation permits the use of more complex models and is thus often used for QRA models to capture the intricacies of a process, but it is fairly slow and inference is not straightforward.

Markov chain Monte Carlo methods offer a good compromise between the BN approach and Monte Carlo simulation in terms of speed and flexibility. They also support inference while being able to accurately model continuous distributions.

Two-dimensional modelling, a method for separating the variability and uncertainty in a model, can be used with MCMC methods and Monte Carlo simulation.

\section{Acknowledgements}

The original case study was funded by the UK Ministry of Agriculture Fisheries and Food, project number FS3305. Subsequent work has been supported under Silsoe Research Institute's Competitive Strategic Grant from the Biotechnology and Biological Sciences Research Council. The authors would also like to thank Professor G. Mead of the Royal Veterinary College, Dr. V. Allen of Bristol University and the poultry companies who were involved in the original study.

\section{References}

Bailey, J.S., Buhr, R.J., Cox, N.A., Berrang, M.E., 1996. Effect of hatching cabinet sanitation treatments on Salmonella cross-contamination and hatchability of broiler eggs. Poultry Sci. 75 (2), $191-196$.

Barker, G.C., Talbot, N.L.C., Peck, M.W., 2002. Risk assessment for Clostridium botulinum: a network approach. Int. Biodeterior. Biodegrad. 50 (3-4), 167-175.

Brewer, R.L., James, W.O., Prucha, J.C., Johnston, R.W., Alvarez, C.A., Kelly, W., Bergeron, E.A., 1995. Poultry-processing line speeds as related to bacteriological profile of broiler carcasses. J. Food Sci. 60 (5), 1022-1024.

Brooks, S., 1998. Markov chain Monte Carlo method and its application. Statistician 47 (1), 69-100.

Carr, L.E., Mallinson, E.T., Tate, C.R., Miller, R.G., Russekcohen, E., Stewart, L.E., Opara, O.O., Joseph, S.W., 1995. Prevalence of Salmonella in broiler flocks - effect of litter water activity, house construction, and watering devices. Avian Dis. 39 (1), 39-44.

Carramiñana, J.J., Yangüela, J., Blanco, D., Rota, C., Agustin, A.I., Ariño, A., Herrera, A., 1997. Salmonella incidence acid distribution of serotypes throughout processing in a Spanish poultry slaughterhouse. J. Food Prot. 60 (11), 1312-1317.

Cason, J.A., Bailey, J.S., Stern, N.J., Whittemore, A.D., Cox, N.A., 1997. Relationship between aerobic bacteria, Salmonellae, and Campylobacter on broiler carcasses. Poultry Sci. 76 (7), 1037-1041.

Cassin, M.H., Lammerding, A.M., Todd, E.C.D., Ross, W., McColl, R.S., 1998. Quantitative risk assessment for Escherichia coli O157:H7 in ground beef hamburgers. Int. J. Food Microbiol. 41 (1), 21-44.

Clouser, C.S., Doores, S., Mast, M.G., Knabel, S.J., 1995. The role of defeathering in the contamination of turkey skin by Salmonella species and Listeria monocytogenes. Poultry Sci. 74 (4), $723-731$.

Davies, R.H., Wray, C., 1995. Mice as carriers of Salmonella enteritidis on persistently infected poultry units. Vet. Rec. 137 (14), $337-341$.

Edel, W., 1994. Salmonella enteritidis eradication program in poultry breeder flocks in the Netherlands. Int. J. Food Microbiol. 21 $(1-2), 171-178$.

Hargis, B.M., Caldwell, D.J., Brewer, R.L., Corrier, D.E., Deloach, J.R., 1995. Evaluation of the chicken crop as a source of Salmonella contamination for broiler carcasses. Poultry Sci. 74 (9), $1548-1552$.

Hoornstra, E., Notermans, S., 2001. Quantitative microbiological risk assessment. Int. J. Food Microbiol. 66, 21-29.

Jensen, F.V., 1996. An Introduction to Bayesian Networks UCL Press, London, UK.

Larsen, G.J., Rolow, A.M., Nelson, C.E., 1993. The effect of organic-acids on Salmonella contamination originating from mouse fecal pellets. Poultry Sci. 72 (9), 1797-1799.

Ramirez, G.A., Sarlin, L.L., Caldwell, D.J., Yezak, C.R., Hume, M.E., Corrier, D.E., Deloach, J.R., Hargis, B.M., 1997. Effect of feed withdrawal on the incidence of Salmonella in the crops and ceca of market age broiler chickens. Poultry Sci. 76 (4), 654-656. 
Royal Society, 1992. Risk: Analysis, Perception and Management. London, UK.

Spiegelhalter, D.J., Thomas, A., Best, N.G., Lunn, D., 2003. WinBUGS Version 1.4 User Manual. MRC Biostatistics Unit, Cambridge, UK.

Vose, D.J., 1998. The application of quantitative risk assessment to microbial food safety. J. Food Prot. 61 (5), 640-648.
Whiting, R.C., Buchanan, R.L., 1997. Development of a quantitative risk assessment model for Salmonella enteritidis in pasteurised liquid eggs. Int. J. Food Microbiol. 36 (2-3), 111-125.

WHO/FAO, 2002. Risk assessments of Salmonella in eggs and broiler chickens. Microbiological Risk Assessment Series No. 2. World Health Organization, Geneva, Switzerland/Food and Agriculture Organization of the United Nations, Rome, Italy. 